\title{
Dynamic Driver Supply Voltage Scaling for Organic Light Emitting Diode Displays
}

\author{
Donghwa Shin, Student Member, IEEE, Younghyun Kim, Student Member, IEEE, \\ Naehyuck Chang, Fellow, IEEE and Massoud Pedram, Fellow, IEEE
}

\begin{abstract}
Organic light emitting diode (OLED) display is a self-illuminating device that is supposed to be power efficient than liquid crystal display (LCD). However, OLED display panels consume as much power as LCD panels due to total internal refection. As the power consumption of the OLED panel depends on the pixel colors, most previous power saving methods alter the pixel colors. In practice, such OLED power saving techniques can hardly accommodate photo viewers and movie players.

This paper introduces the first OLED power saving technique that dynamically changes the supply voltage of the panel. Reduced supply voltage results in both power saving and decreased pixel luminance, but model-based color correction restores the decreased luminance with minimum color distortion. This technique is similar to dynamic backlight scaling of LCDs but is based on the unique characteristics of the OLED drivers. We provide an online color compensation algorithm using the luminance histogram. Luminance quantization in the histogram also achieves resource minimization. We develop a prototype and demonstrate the proposed OLED dynamic voltage scaling (DVS). Experimental result shows that the proposed OLED DVS saves up to $74.7 \%$ of the display power for the still images and up to $35.9 \%$ for movie clips.
\end{abstract}

\section{INTRODUCTION}

D ISPLAY systems are primary sources of power consumption in battery-powered electronics despite the advances in low-power display technologies. As of today, liquid crystal display (LCD) panels are widely used in portable as well as desktop systems. The LCD panels do not illuminate themselves and require a high intensity backlight, which generally consumes a significant amount of power due to low transmittance of the LCD panels [2], [3]. On the other hand, an organic light emitting diode (OLED) is self-illuminating using organic light emission material. Therefore, OLEDs should provide higher brightness, higher luminance, faster response, wider viewing angle, and thinner and lighter-weight form factors compared with conventional LCD panels [4].

This work is supported from the Brain Korea 21 Project, IC Design Education Center (IDEC), and Mid-career Researcher Program through NRF grant funded by the MEST (No. 2010-0017680). The ICT at Seoul National University provides research facilities.

This paper was presented in part at the 2011 Design Automation Conference (DAC), San Diego, USA [1].

D. Shin, Y. Kim, and N. Chang are with the Department of Electrical Engineering and Computer Science, Seoul National University, Seoul 151-742, Korea (e-mail: dhshin@elpl.snu.ac.kr; jhpark@elpl.snu.ac.kr; naehyuck@elpl. snu.ac.kr).

M. Pedram is with the Department of Electrical and Electronic Engineering, University of Southern California, Los Angeles, CA, USA (e-mail: pedram@usc.edu).

Paper Number: ...

Digital Object Identifier:
One of the known major disadvantages of OLED panels was their relatively short lifetime, which has been enhanced to be commercialized. However, the power efficiency of the OLED panels is not as high as expected due to serious total internal refection. As a result, most OLED smartphone users do not really feel extended battery life from the OLED display.

There have been extensive efforts to reduce the OLED panel power consumption. Most previous work attempted aggressive dimming of a part of the panel to reduce power consumption because the OLED power consumption is directly dependent on the pixel color. As red, green, and blue colors show distinctly different power efficiency, color swapping was also proposed. We summarize the previous work in Section II.

In this paper, we introduce OLED dynamic voltage scaling (DVS). We dynamically change the supply (driving) voltage of the OLED panel and save power. The proposed technique exploits the unique characteristics of the OLED driver circuits. The supply voltage of an OLED driver circuit is set to high enough voltage to support the full luminance of a pixel. An excessive voltage is generally supplied to the most pixels for most of the time, which gives headroom to apply supply voltage scaling. However, we cannot avoid minor pixel distortion because all the pixels have one power supply source, and pixel-level DVS is not allowed. Reduced supply voltage alters the pixel color value even with non-negative headroom. We restore the original pixel color by the use of model-based color compensation method.

We summarize major technical contributions of this paper as follows:

- Development of the concept of DVS for the OLED displays.

- Power analysis and modeling of OLED display.

- Development of the OLED DVS model.

- Image compensation algorithm that minimizes pixel color distortion after DVS.

- Online method based on the luminance histogram and histogram optimization by luminance quantization.

- Prototype implementation.

The rest of the paper is organized as follows. Section II summarizes previous research on low-power techniques for the display system. Section III introduces characteristics of the OLED cell, driver circuits and the principles of operation of the OLED DVS. Section IV presents a power optimization algorithm while maintaining the image distortion within a given threshold. Section V presents an online OLED DVS method based on the luminance histogram and its optimiza- 
TABLE I

CLASSIFICATION OF DISPLAY POWER SAVING TECHNIQUES.

\begin{tabular}{|c|c|c|c|}
\hline Techniques & Features & Applications & Displays \\
\hline \hline $\begin{array}{c}\text { Usage behavior } \\
\text { monitoring }\end{array}$ & $\begin{array}{c}\text { Not functional during } \\
\text { low-power mode }\end{array}$ & $\begin{array}{c}\text { Interactive } \\
\text { applications }\end{array}$ & $\begin{array}{c}\text { CRT, LCD } \\
\text { and OLED }\end{array}$ \\
\hline $\begin{array}{c}\text { Partial display } \\
\text { turn off }\end{array}$ & $\begin{array}{c}\text { Disable objects } \\
\text { not in interest }\end{array}$ & $\begin{array}{c}\text { Mixed active } \\
\text { /idle objects }\end{array}$ & $\begin{array}{c}\text { LCD* } \\
\text { and OLED }\end{array}$ \\
\hline $\begin{array}{c}\text { Color } \\
\text { remapping }\end{array}$ & $\begin{array}{c}\text { Altered look } \\
\text { and feel }\end{array}$ & GUI & $\begin{array}{c}\text { LCD } \\
\text { and OLED }\end{array}$ \\
\hline $\begin{array}{c}\text { Backlight } \\
\text { scaling }\end{array}$ & $\begin{array}{c}\text { Minor color } \\
\text { distortion }\end{array}$ & $\begin{array}{c}\text { No } \\
\text { restrictions }\end{array}$ & LCD \\
\hline
\end{tabular}

*LCD panels with zoned backlighting.

tion. Section VI introduces the prototype implementation, and Sections VII presents the experimental results.

\section{RELATED WORK}

One of the simplest display power saving methods is brightness dimming or timeout-based display turnoff, which is actually widely used for most commercial products. Such a method can save display power at the direct expense of inconvenience. On the other hand, intensive research has been made to minimize user inconvenience as summarized in Table I. The first two categories of techniques disable the display functionality of the entire panel or part of the panel whereas the last two categories of techniques apply a transformation to the image being displayed.

Most high-level power saving methods are based on idleness of the system. Display does not generally have idleness while it is turned on. Nevertheless, first category methods actively detect idleness of the display detecting human eye gaze by the use of a camera [5], [6]. These techniques are applicable to any types of display with an interactive application where the user does not always pay attention to the display.

The second category of techniques is dedicated to LCD with zoned backlighting and OLED displays that allow partial display turnoff [7], [8]. This method is useful for distinguishable foreground and background objects such as multiple windows operating systems. Only active foreground objects have the original backlight luminance and other background or inactive objects have backlight turned off or dimmed [7]. OLED panel can freely change the colors of objects that are out of focus to dark colors [8].

The third category of techniques is also dedicated to LCD and OLED displays. They attempt content (color) change of the displayed image exploiting the power consumption difference by the pixel colors [9], [10], [11]. LCD panels exhibit around $10 \%$ power consumption difference due to change in the colors being displayed [12]. In addition, pixel color remapping provides more headroom for backlight dimming and, in turn, higher power saving [9]. Color remapping also has a big impact on the OLED panel power consumption [10]. Unfortunately, color remapping is not always feasible. It is applicable only to the graphics user interface (GUI) and applications not dealing with natural images, photos, or video.

Techniques in the last category reduce the backlight luminance and adjust colors to enhance the brightness and/or contrast of the image to compensate the image quality degrada-

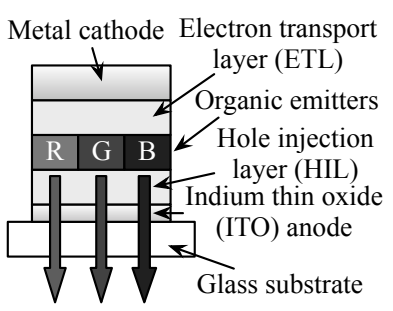

(a) OLED device structure

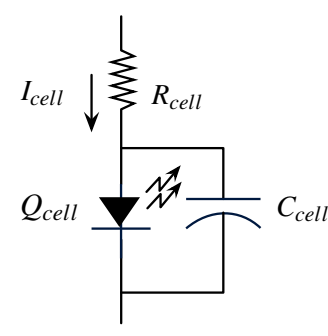

(b) Equivalent circuit model
Fig. 1. Device structure of OLED (a) and equivalent circuit model (b).

tion [2], [3], [13], [14], and [15]. These backlight scaling techniques do not incur noticeable image degradation, nor does it result in a large color change. The LCD backlight scaling is widely used for commercial products nowadays.

Unfortunately, backlight scaling cannot be applicable to self-illuminating display devices such as the OLED panels. Recent work introduces supply voltage scaling of the OLED derivers [1]. This method i) induces only minimal color change to accommodate display of natural images and ii) is applicable to only the displayed object that is of interest to the users. As the power consumption of an OLED panel is dependent on each pixel color value, existing OLED power management techniques are not capable of altering power consumption of the OLED panel while keeping the pixel color values. Follow-up research introduces a DVS-friendly OLED driver circuit design [16]. It maintains the cell current when the supply voltage scales by adding additional transistor on the gate side of the driver transistor. This paper is an extension of the basic OLED DVS presented in [1].

\section{SUPPly VOLTAGE SCALING OF OLED DRIVERS}

\section{A. OLED display structures and driver circuits}

Fig. 1(a) shows the typical structure of the OLED cell [4]. The OLED device has a large area, but the thickness of the organic layers between the electrodes is only 100-200 nm. As a result, OLED cells have a large internal capacitance. The value of $C_{\text {cell }}$ is typically $200-400 \mathrm{pF} / \mathrm{mm}^{2}$. OLED cells have a resistive component for each layer that lies between anode and cathode. An Indium-Thin-Oxide (ITO) layer is the dominant resistive component. Hence, the parasitic resistor is in series with the internal capacitance. The value of the parasitic resistor is strongly dependent on the design of the ITO electrode (anode). A typical value of the cell resistance is $15 \Omega / \mathrm{sq}^{1}$. We calculate the $R_{\text {cell }}$ with the cell area and sheet resistance. A simple equivalent circuit obtained with the physical parameters is depicted in Fig. 1(b). It consists of the parasitic resistor $R_{\text {cell }}$, internal capacitance $C_{\text {cell }}$, and a diode $Q_{\text {cell }}$.

There are several ways to classify the OLED driver architectures. Like LCD panels, an OLED panel may have either a passive matrix (PMOLED) or an active matrix (AMOLED) structures. A PMOLED panel has simpler structure and thus lowers cost. However, the practical maximum size is limited, typically up to 3". PMOLED panels have a row-column

\footnotetext{
${ }^{1} \Omega /$ sq denotes the sheet resistance.
} 


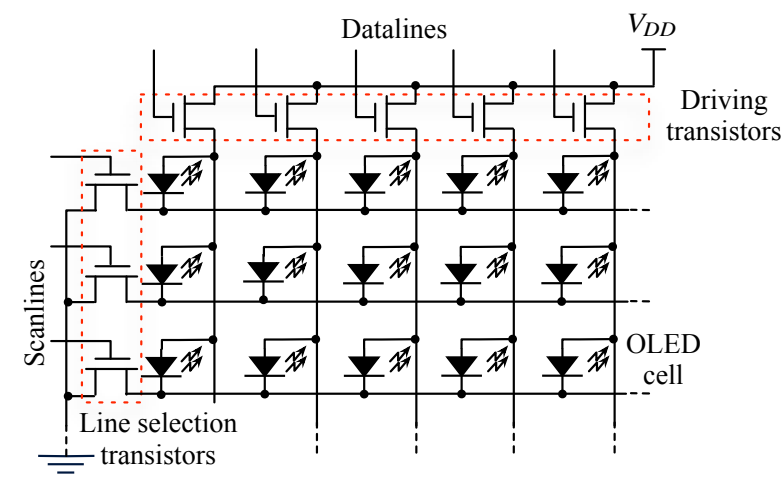

(a) Passive-matrix OLED display

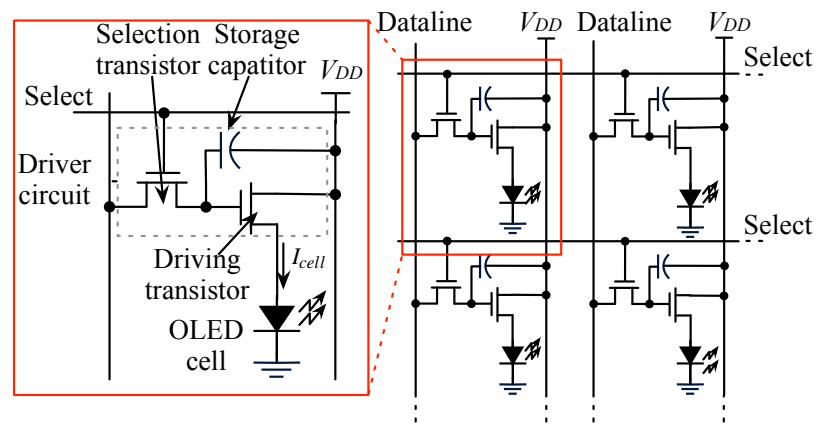

(b) Active-matrix OLED display

Fig. 2. Panel structures of OLED displays: (b) active-matrix OLED display and (a) passive-matrix OLED display.

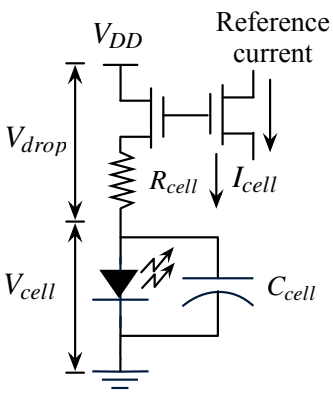

(a) AM driver

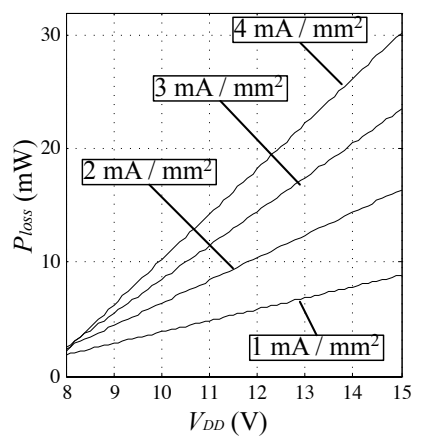

(b) SPICE simulation result
Fig. 3. Behavioral concept of (a) AM driver and (b) SPICE simulation result of $P_{\text {loss }}$ with different $V_{D D}$ and $I_{\text {cell }}$ in AM driver circuit.

structure driver circuit as shown in Fig. 2(a). There is no storage capacitor in the PMOLED driver circuit. The cell current can be a pulsed current. In contrast, a thin film transistor (TFT) with a storage capacitor controls every pixel of AMOLED panels similar to the TFT LCD panel as shown in Fig. 2(b). AMOLED panels can accommodate large size and high fidelity display.

The OLED cell current, $I_{\text {cell }}$, determines the luminance. The cell current is basically controllable by adjusting the cell voltage, $V_{\text {cell }}$. However, we commonly use a constant current driver because the parasitic resistance is not stable. A current mirror current steering circuit forms an amplitude modulation (AM) driver. The AM driver scheme ensures a higher reliability and efficiency of the OLED cells. The current steering circuit consumes large area, which results in higher cost.

An alternative way of current control is a pulse width modulation (PWM) of the cell current, which is common in the PMOLED panels. The luminance of an OLED cell is dependent on the average value of $I_{\text {cell }}$. The PWM cell current steering circuit is inexpensive and provides precise luminance control. However, it is known to be less power efficient in high luminance region [4].

\section{B. $V_{D D}$ scaling for $A M$ drivers}

The concept of DVS of an OLED panel is to reduce power loss due to $V_{d r o p}$ by scaling down $V_{D D}$. Although we scale

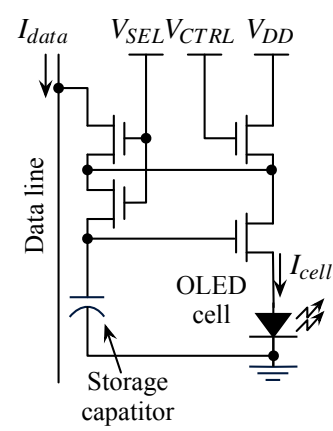

Fig. 4. DVS-friendly AM driver circuit [16].

down the $V_{D D}$ of the AM driver circuit, there is only small change in $I_{\text {cell }}$ due to the Early Effect in the AM driver as far as the driving transistor remains in the saturation mode (Fig. 3(a)). The driving transistor enters the triode mode when $I_{c e l l}$ becomes too large with the scaled $V_{D D}$. The cell luminance decreases as we scale down $V_{D D}$ in the triode mode, which causes image distortion.

The power loss of OLED cell is defined by $P_{\text {loss }}=I_{\text {cell }} V_{\text {drop }}$ where $V_{d r o p}$ is determined by the characteristics of the OLED cell and $I_{\text {cell }}$ is determined by the saturation current of the driver transistor. The $V_{D D}$ should be dropped by power dissipation in the driver transistor, and $P_{\text {loss }}$ due to $V_{d r o p}$ is given by

$$
P_{\text {loss }}=I_{\text {cell }} V_{\text {drop }}=I_{\text {cell }}\left(V_{D D}-V_{f}\right),
$$

where $V_{f}$ is the forward bias voltage of the diode.

Fig. 3(b) shows a SPICE simulation to estimate $P_{\text {loss }}$ with the parameters from [4]. The simulation model has V-I characteristics as follows:

$$
I_{\text {cell }}=1.4144 \cdot 10^{-6}\left(e^{\frac{V_{\text {cell }}}{0.93678}}-1\right),
$$

with $20 \mathrm{~mm}^{2}$ active area. We estimate $P_{\text {loss }}$ with various $V_{D D}$ values while delivering four different $I_{c e l l}$ values from $1 \mathrm{~mA} / \mathrm{mm}^{2}$ to $4 \mathrm{~mA} / \mathrm{mm}^{2}$. The simulation result depicted in Fig. 3 (b) shows that $P_{\text {loss }}$ is proportional to the $V_{D D}$ and $I_{c e l l}$ as described in (1).

Fig 4 depicts a DVS-friendly OLED driver circuit that is more amenable to supply voltage scaling [16]. The DVSfriendly driver makes color distortion happen only when $V_{D D}$ is too low to supply $I_{\text {cell }}=I_{\text {data }}$ because the bias condition 


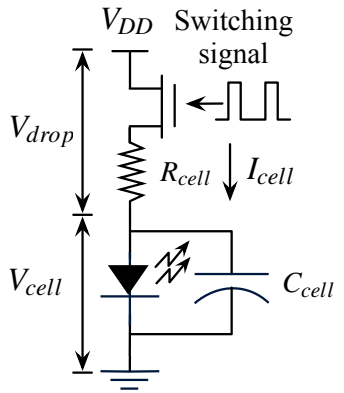

(a) PWM driver

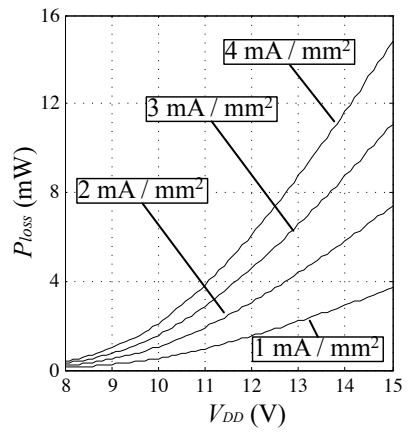

(b) SPICE simulation result
Fig. 5. Behavioral concept of (a) PWM driver and (b) SPICE simulation result of $P_{\text {loss }}$ with various $V_{D D}$ and $I_{\text {cell }}$ values.

of $T_{4}$ is maintained by storage capacitor and $V_{c t r l}$. So, $I_{\text {cell }}$ is equal to $I_{\text {data }}$ as long as the $V_{D D}$ is high enough. The DVS-friendly AM driver makes OLED DVS more efficient.

\section{C. $V_{D D}$ scaling for $P W M$ drivers}

DVS acts a bit differently in a PWM driver (Fig. 5 (a)). Scaling $V_{D D}$ down directly affects $I_{\text {cell }}$. We have to restore the luminance of image even with a slight $V_{D D}$ scale. We apply model-based image compensation and restore the luminance. A brighter color makes a higher PWM duty ratio in the PWM driver. The image compensation cannot always restore the original luminance if the original $I_{c e l l}$ is too large. The maximum possible $I_{c e l l}$ under the scaled $V_{D D}$ cannot be the same as the original $I_{c e l l}$ even when the PWM duty ratio is set to $100 \%$. Thus, luminance distortion for some very bright pixels becomes unavoidable. We sacrifice a small display quality by allowing a certain amount of color distortion of the image but save significant amount of power consumption.

With the PWM drivers, $V_{f}$ and $R_{c e l l}$ determine the maximum value of $I_{\text {cell }}$ as follows:

$$
I_{c e l l}=\frac{V_{D D}-V_{f}}{R_{\text {cell }}} .
$$

The luminance of the OLED is approximately proportional to the average value of $I_{c e l l}, \overline{I_{c e l l}}$, which is calculated by

$$
\overline{I_{c e l l}}=I_{c e l l} d=I_{c e l l} \frac{t_{\text {on }}}{t_{\text {on }}+t_{\text {off }}},
$$

where PWM duty, $d=t_{o n} /\left(t_{o n}+t_{o f f}\right)$, and $t_{\text {on }}$ and $t_{\text {off }}$ are the switch turn on and off durations in a PWM period, respectively. The power loss of an OLED cell during a PWM period is given by

$$
P_{\text {loss }}={\overline{I_{\text {cell }}}}^{2} R_{\text {cell }}
$$

Fig. 5 (b) shows a SPICE simulation result to estimate $P_{\text {loss }}$ of the PWM driver such that $P_{\text {loss }}$ quadratically increases as the $V_{D D}$ and $I_{\text {cell }}$ increase as described in (4). We use the same simulation parameters in Section III-B. We estimate $P_{\text {loss }}$ with various $V_{D D}$ while delivering four different $I_{\text {cell }}$ values from $1 \mathrm{~mA} / \mathrm{mm}^{2}$ to $4 \mathrm{~mA} / \mathrm{mm}^{2}$.

\section{POWER OPTIMIZATION CONSIDERING IMAGE QUALITY}

\section{A. Image quality and power models of OLED panels}

We use human perception-aware color model to evaluate the image distortion. Typical RGB and CMYK spaces reflect the output of physical devices rather than human visual perception. CIE Lab color space is designed to approximate human-perceived vision. It is derived from the CIE 1931 $\mathrm{XYZ}$ color space, which reflects the spectral distribution of colors, and can be computed via simple formulas from the XYZ space. Due to its perceptual uniformity, its $L$ component closely matches the human perception of brightness. The Euclidean distance in the Lab color space is widely used as a metric to measure the human perceived color difference [17].

The XYZ measurement result shows that $X, Y$, and $Z$ values of RGB pixels are highly correlated (almost linearly proportional) with the cell current or almost constant regardless of the cell current. We build a transformation function using regression analysis, which is given by

$$
\left[\begin{array}{l}
X \\
Y \\
Z
\end{array}\right]=\left[\begin{array}{l}
a_{X} \\
a_{Y} \\
a_{Z}
\end{array}\right] I_{\text {cell }}+\left[\begin{array}{c}
b_{X} \\
b_{Y} \\
b_{Z}
\end{array}\right],
$$

where coefficients $a_{X}, a_{Y}, a_{Z}, b_{X}, b_{Y}$, and $b_{Z}$ are obtained by performing the regression analysis on the measurement results.

We construct an $I_{\text {cell }}$ model for a PMOLED panel with a PWM driver based on (2) and (3). The cell current $I_{c e l l}$ is proportional to $V_{D D}$ and $d$ such that

$$
I_{c e l l}\left(d, V_{D D}\right)=p_{1} V_{D D} d+p_{2} d+p_{3},
$$

where $p_{1}, p_{2}$, and $p_{3}$ are characteristic coefficients.

We describe the human-perceived image difference with the Euclidean distance in the CIE Lab color space. We transform $I_{x y z}=(X, Y, Z)$ into a Lab color space image such that $I_{l a b}=$ $(L, a, b)$ by using the following transform functions [18].

$$
\begin{aligned}
& L=116 \cdot\left(Y / Y_{w}\right)^{\frac{1}{3}}-16 \\
& a=500 \cdot\left(\left(X / X_{w}\right)^{\frac{1}{3}}-\left(Y / Y_{w}\right)^{\frac{1}{3}}\right) \\
& b=200 \cdot\left(\left(Y / Y_{w}\right)^{\frac{1}{3}}-\left(Z / Z_{w}\right)^{\frac{1}{3}}\right),
\end{aligned}
$$

where $L, a$ and $b$ are matrices representing brightness, redgreen content, and yellow-blue content in the Lab color space, respectively. Values of $X_{w}, Y_{w}$, and $Z_{w}$ are the color coordinate values of the reference white in the color space. The Euclidean distance between two different colors $c_{1}=\left(L_{1}, a_{1}, b_{1}\right), c_{2}=$ $\left(L_{2}, a_{2}, b_{2}\right)$ in the Lab color space is calculated by

$$
\varepsilon=\sqrt{\left(L_{1}-L_{2}\right)^{2}+\left(a_{1}-a_{2}\right)^{2}+\left(b_{1}-b_{2}\right)^{2}} .
$$

\section{B. OLED display characterization}

We chose a target OLED panel from Univision Technology [19], UG-2076GDEAF02, that has a 2.2" display area, a $220 \times 176$ resolution and a PMOLED structure with a PWM driver. We measure the relationship between the power consumption and luminance/chromaticity of the OLED panel with various $V_{D D}$ values and pixel colors. We setup the measurement environment as shown in Fig. 6. We control $V_{D D}$ with a 


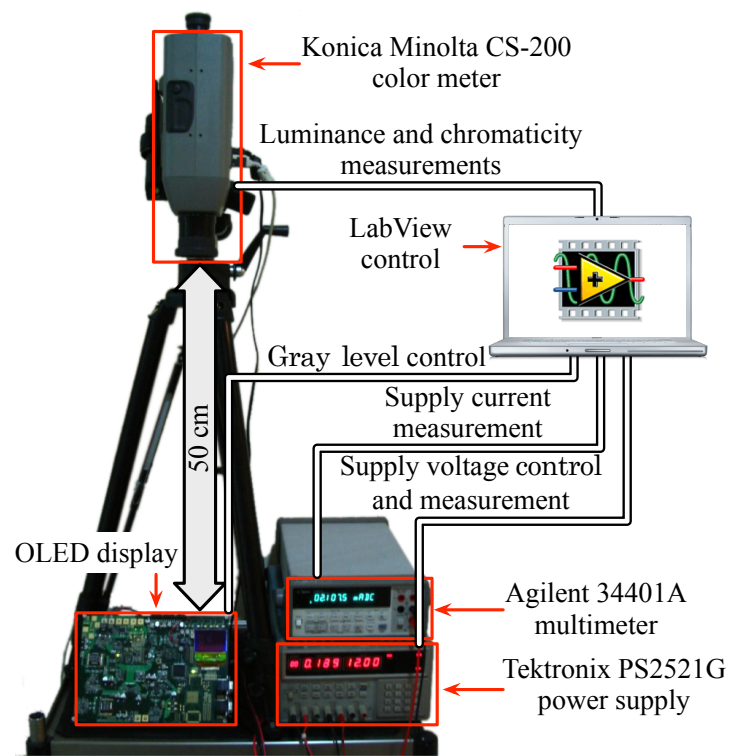

Fig. 6. Experimental setup for the OLED display panel characterization.

programmable power supply and measure the current with an Agilent 24401A digital multimeter. We use a Konica Minolta CS-200 color meter to measure the luminance and chromaticity of the OLED panel. The experiment is automated by using a National Instruments LabView console. We perform the entire measurement process in a darkroom to block the effect of ambient light. We acquire the coefficients by measurement and summarize them in Table II. They show that the OLED cell of the UG-2076 OLED display panel has approximately $15 \Omega$ of $R_{\text {cell }}$ and $7.4 \mathrm{~V}$ of $V_{f}$.

We visualize a part of characterization data in Fig. 7. The OLED display achieves the same luminance by adjusting the color value (gray level here) even with different $V_{D D}$ levels. In other words, we can restore the color value with even a reduced $V_{D D}$, which proves the key premise of DVS for OLEDs. Fig. 7 shows that the OLED panel generates a $70 \mathrm{~cd} / \mathrm{mm}^{2}$ luminance with a $15 \mathrm{~V}$, a $13 \mathrm{~V}$, a $11 \mathrm{~V}$, and a $9 \mathrm{~V} V_{D D}$ by setting the gray level to $57 \%, 59 \%, 64 \%$, and $77 \%$, respectively. It turns out that the luminance is not affected by $V_{D D}$ when the gray level is below a certain level such as non-linear region in Fig. 7. Therefore, we compensate the $V_{D D}$ scaling-induced luminance reduction by modifying image data only in the linear region of Fig. 7.

TABLE II

EXTRACTED PARAMETERS FOR THE POWER ESTIMATION AND IMAGE DIFFERENCE EVALUATION ( $I_{\text {cell }}$ IS IN $\left.\mu \mathrm{A}\right)$.

\begin{tabular}{|c|c|r|r|r|r|r|}
\hline & \multicolumn{2}{|c|}{$\mathrm{R}$} & \multicolumn{2}{|c|}{$\mathrm{G}$} & \multicolumn{2}{c|}{$\mathrm{B}$} \\
\hline \multirow{3}{*}{$\begin{array}{c}\text { I cell } \\
\text { estimation }\end{array}$} & $p_{1}$ & $2.222 \mathrm{e}-2$ & $p_{1}$ & $2.234 \mathrm{e}-2$ & $p_{1}$ & $2.245 \mathrm{e}-2$ \\
\cline { 2 - 7 } & $p_{2}$ & $-1.650 \mathrm{e}-1$ & $p_{2}$ & $-1.648 \mathrm{e}-1$ & $p_{2}$ & $-1.599 \mathrm{e}-1$ \\
\cline { 2 - 7 } & $p_{3}$ & $1.652 \mathrm{e}$ & $p_{3}$ & $1.664 \mathrm{e} 0$ & $p_{3}$ & $1.597 \mathrm{e} 0$ \\
\hline \multirow{4}{*}{$\begin{array}{c}\text { Image } \\
\text { differ- } \\
\text { ence } \\
\text { evaluation }\end{array}$} & $a_{X}$ & $3.573 \mathrm{e} 5$ & $a_{X}$ & $1.035 \mathrm{e} 5$ & $a_{X}$ & $4.903 \mathrm{e} 4$ \\
\cline { 2 - 7 } & $b_{X}$ & $-4.554 \mathrm{e}-1$ & $b_{X}$ & $-2.764 \mathrm{e}-1$ & $b_{X}$ & $-3.230 \mathrm{e}-1$ \\
\cline { 2 - 7 } & $a_{Y}$ & $1.793 \mathrm{e} 5$ & $a_{Y}$ & $2.556 \mathrm{e} 5$ & $a_{Y}$ & $6.139 \mathrm{e} 4$ \\
\cline { 2 - 7 } & $b_{Y}$ & $-2.282 \mathrm{e}-1$ & $b_{Y}$ & $-7.086 \mathrm{e}-1$ & $b_{Y}$ & $-3.020 \mathrm{e}-1$ \\
\cline { 2 - 7 } & $a_{Z}$ & $0.000 \mathrm{e} 0$ & $a_{Z}$ & $2.263 \mathrm{e} 4$ & $a_{Z}$ & $2.384 \mathrm{e} 5$ \\
\cline { 2 - 7 } & $b_{Z}$ & $7.100 \mathrm{e}-3$ & $b_{Z}$ & $-6.030 \mathrm{e}-2$ & $b_{Z}$ & $-1.937 \mathrm{e} 1$ \\
\hline
\end{tabular}

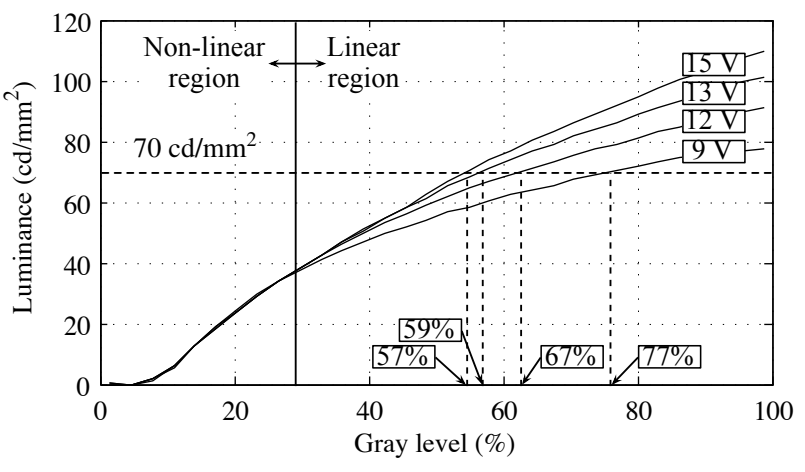

Fig. 7. Measured luminance by $V_{D D}$ and gray level with AM driver.

\section{C. $V_{D D}$ scaling and image compensation}

The transistor in the AM driver is originally designed to operate in the saturation mode. The driver transistor is in the saturation mode though we scale $V_{D D}$ as shown in Fig. 8 as long as $V_{D S}$ is higher than $V_{G S}-V_{T}$. The saturation mode operation ensures the almost same $I_{\text {cell }}$ regardless of changes in the $V_{D D}$. There is only small change of $I_{\text {cell }}$ due to the Early effect. Consequently, $V_{D D}$ scaling only affects pixels with high brightness as shown in Fig. 8. High brightness pixels can no longer deliver the same amount of the cell current with a reduced $V_{D D}$. We need to limit the number of distorted pixels to maintain the image quality, and $V_{D D}$ should be determined under the considerations of the upper bound of the distorted pixels.

We have more potential to save power consumption from $V_{D D}$ scaling with the PWM drivers. We reduce $P_{\text {loss }}$ in an OLED cell while preserving the luminance in spite of a reduced $V_{D D}$ by raising $d$ according to (2), (3), and (4). The scaled $V_{D D}$ for PWM drivers evenly decreases the luminance of all the OLED cells. At the same time, we restore $\overline{I_{\text {cell }}}$ and the luminance by increasing the PWM duty ratio $d$ in (3) as shown in Fig. 9. Then we obtain the same $\overline{I_{\text {cell }}}$ with less $P_{\text {loss }}$.

The CIE Lab color space regards two different colors perceptually identical when the Euclidean difference between the two color points is less than a certain threshold. The threshold is generally determined by the human vision characteristics and environmental conditions, and the user also can determine it. We formulate an optimization problem

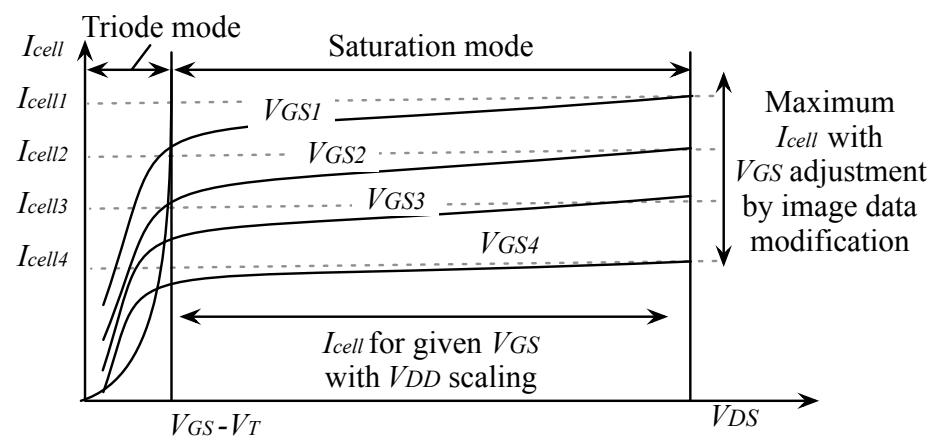

Fig. 8. Effect of $V_{D D}$ scaling and image compensation on the OLED cell current with AM drivers. 

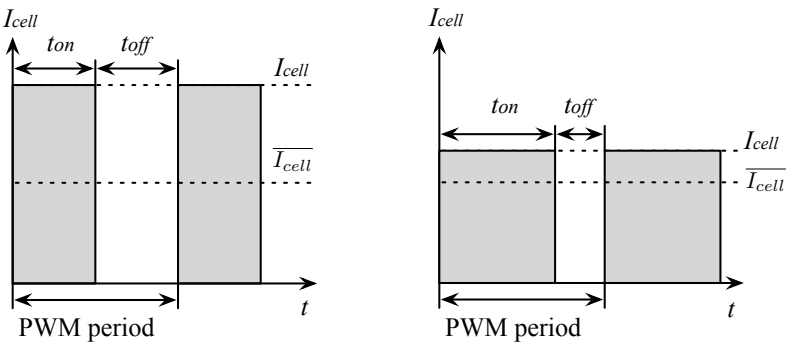

(a) OLED cell current with the maximum (b) OLED cell current with the scaled $V_{D D}$ $V_{D D}$

Fig. 9. Effect of $V_{D D}$ scaling and image data modification on OLED cell current with (a) the maximum $V_{D D}$ and (b) scaled $V_{D D}$ for PWM drivers.

to find a transformed image $I^{\prime}=\left(R^{\prime}, G^{\prime}, B^{\prime}\right)$ and $V_{D D}$ that maximize the power saving subject to the given threshold for indiscernible colors. The threshold, $\tau_{\text {image }}$, can be thought of as the maximum allowable average Euclidean distance $\bar{\varepsilon}$ between the original and compensated images. We develop an iterative algorithm to find the solution with image quality and power model of the OLED display as shown in Algorithm1.

Fig. 10 illustrates the behavior of the OLED DVS algorithm with the OLED panel. Upper surface plot of Fig. 10 shows the OLED panel power consumption and lower surface plot shows the luminance value according to the gray level of pixels and $V_{D D}$. The 'Original' dot in the Fig. 10 represents the original $V_{D D}$ and gray level. The dot moves straight down by $V_{D D}$ scaling ((a) in Fig. 10), losing luminance and consuming less power. The image compensation ((b) in Fig. 10) recovers the luminance with a higher gray-level value. This new graylevel incurs higher power consumption, but the 'Scaled' dot eventually exhibits lower power consumption than that of the 'Original' dot while having the same luminance. As the available voltage levels are limited desecrate values, $V_{D D}$ and gray

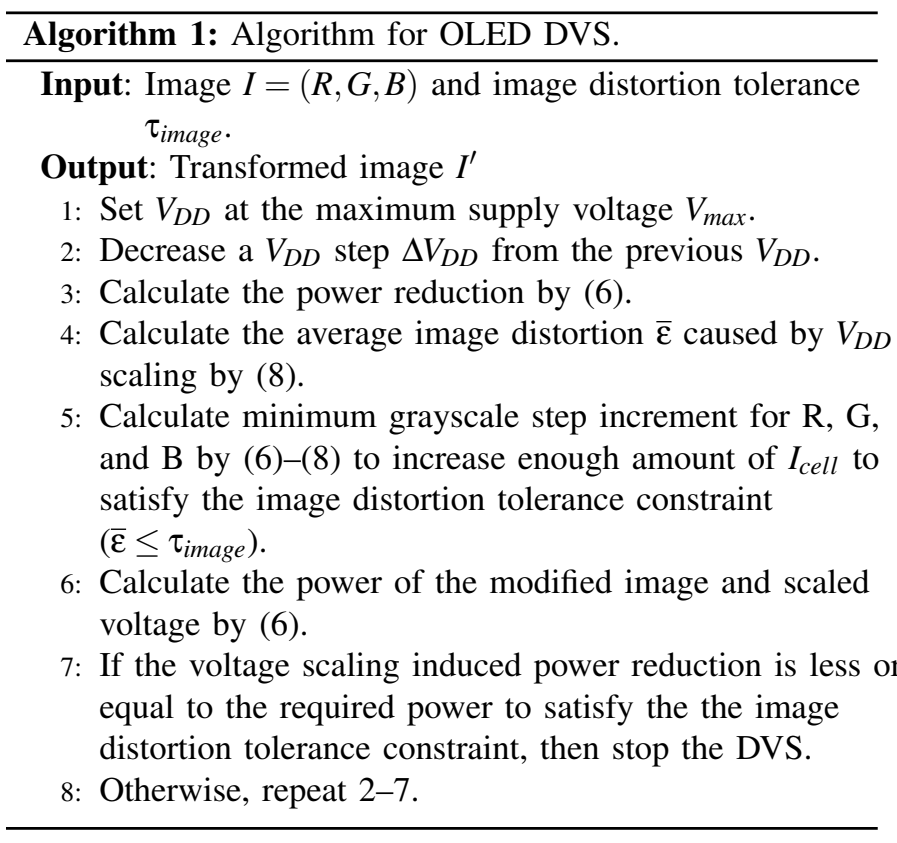

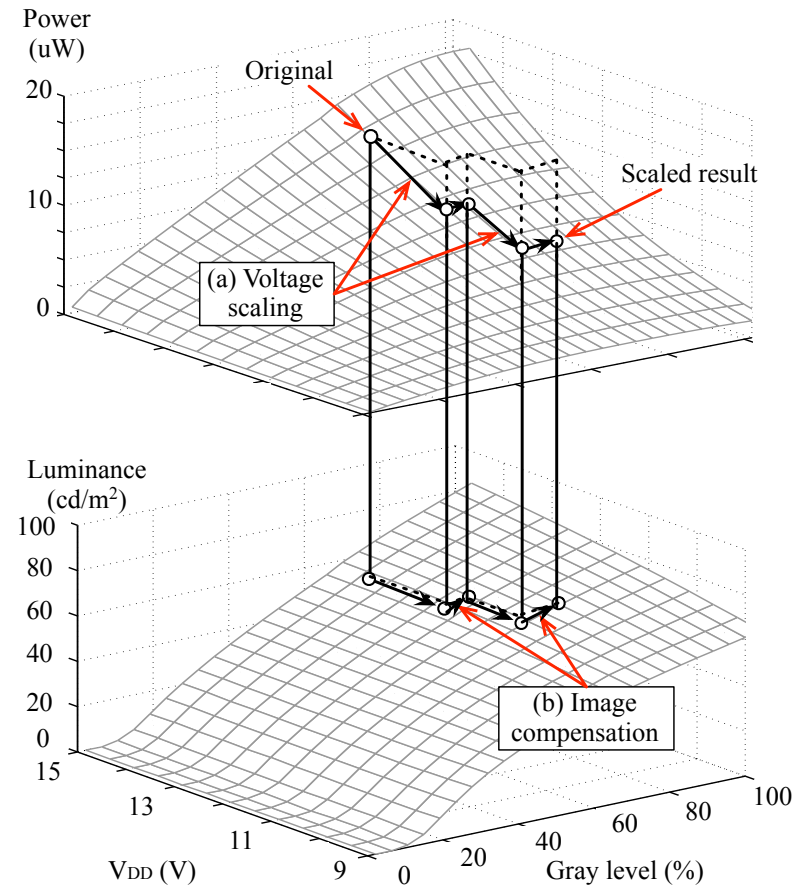

Fig. 10. Power and luminance measurement with a different $V_{D D}$ and image data.

level are discrete, too. Algorithm 1 depicts how to iteratively derive the optimal discrete $V_{D D}$ and gray scale level.

The major computational overhead of OLED DVS is the estimation of the image distortion and calculation of image compensation. We derive them by using a pre-generated lookup table depending on the characteristics of the OLED panel and the driver architecture [2]. The number of color values and $V_{D D}$ levels determine the table size. These parameters strongly affect the performance obtained by the proposed OLED DVS scheme such as delay penalty to display/update an image on the OLED panel and power saving. We introduce a design optimization of the OLED DVS system considering power, image quality, and required resources in Section V.

\section{HARDWARE SUPPORT FOR ONLINE OLED DVS}

We design hardware support unit for an online OLED DVS control. We use pixel luminance histogram and build a lookup table with the scaled $V_{D D}$ as the input and estimates of the image distortion as the output. The estimation identifies pixels having higher luminance value that cannot be produced with the scaled $V_{D D}$ even after image compensation.

We count the number of pixels from the brightest one to limit the image distortion within $\tau_{\text {image }}$. More precise characterization result is obtained with smaller intervals in the histogram [3]. We estimate the side effect in power saving from the histogram quantization interval and derive the most efficient quantization granularity.

\section{A. Estimation of image distortion}

Fig. 11 shows how to derive the threshold luminance $L_{T}$ as a function of $d$ and $V_{D D}$ from (5), (6), and (7). The luminance 


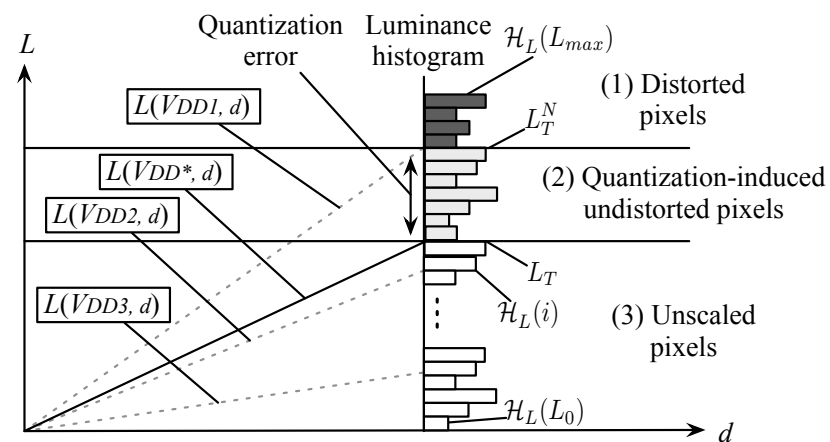

Fig. 11. Side effect in power saving due to the quantization in the histogram.

threshold $L_{T}$ represents an upper limit of luminance that is not distorted by the $V_{D D}$ scaling. The highest possible luminance value without $V_{D D}$ scaling is $L_{\max }$. We define $L_{T}$ corresponding to the image distortion threshold $\tau_{\text {image }}$ by the following condition:

$$
\sum_{i=L_{T}}^{L_{\max }} D(i) \mathcal{H}_{L}(i) \leq \tau_{\text {image }} \text { and } \sum_{i=L_{T}-1}^{L_{\max }} D(i) \mathcal{H}_{L}(i)>\tau_{\text {image }}
$$

where $\mathcal{H}_{L}(i)$ represent the number of pixels whose luminance value is $i$, and $D(i)$ represents the corresponding distortion value. Pixels with the luminance higher than $L_{T}$ (Fig. 11 (1) and (2)) will be distorted even with a histogram without quantization. OLED DVS reduces $P_{\text {loss }}$ by scaling $V_{D D}$ to $V_{D D}^{*}$ corresponding $L_{T}$ where $V_{D D}^{*}$ denotes required voltage level to illuminate the pixels at $L_{T}$ with the maximum $d$ value. Pixels with the luminance lower than $L_{T}$ (Fig. 11 (3)) are restored by the image compensation.

Histogram quantization in image compensation [3] affects the upper limit of luminance that is not distorted by the $V_{D D}$ scaling. Let us denote the luminance value with a quantized $N$-step histogram as $L_{T}^{N}$. Quantization error makes $L_{T} \leq L_{T}^{N}$.

$$
L_{T}^{N}=\left\lceil\frac{L_{T}}{\left(L_{\max } / n\right)}\right\rceil \frac{L_{\max }}{n} .
$$

Pixels with the luminance higher than $L_{T}^{N}$ (Fig. 11 (1)) are distorted because the luminance value should be mapped to $L_{T}^{N}$ while pixels in (Fig. 11 (2)) are not distorted. However, less pixel distortion implies less power saving.

We analyze the amount of power saving sacrificed due to histogram quantization by the interval step, $N$. This is a guidance to obtain the optimal design of image analyzer considering accuracy and overhead. The difference between $P_{l o s s}$ and $\Delta P_{\text {loss }}$ by $V_{D D}$ and $V_{D D}^{\prime}$ is power saving, which is given by

$$
\begin{aligned}
& \Delta P_{\text {loss }}\left(V_{D D}, V_{D D}^{\prime}, d\right) \\
& =R_{\text {cell }}\left[\left(\frac{V_{D D}-V_{f}}{R_{\text {cell }}}\right)^{2} \cdot d-\left(\frac{V_{D D}^{\prime}-V_{f}}{R_{\text {cell }}}\right)^{2} \cdot d^{\prime}\right], \\
& =R_{\text {cell }} \frac{V_{D D}-V_{f}}{R_{\text {cell }}} \cdot\left(V_{D D}-V_{D D}^{\prime}\right) \cdot d
\end{aligned}
$$

where $d^{\prime}$ is determined by following equation to supply the same average $I_{c e l l}$ with $V_{D D}$ and $V_{D D}^{\prime}$.

$$
d^{\prime}=\frac{V_{D D}-V_{f}}{V_{D D}^{\prime}-V_{f}} \cdot d
$$

We calculate the difference of $P_{\text {loss }}$ between the histogram with and without $N$-step quantization by $V_{D D}^{*}$ and $V_{D D}^{* N}$ that correspond to $V_{D D}$ for $L_{T}$ and $L_{T}^{N}$, respectively. We denote required voltage level to illuminate the pixels as $L_{T}^{N}$ with the maximum $d$ value by $V_{D D}^{*}$. Expectation of the difference between $\Delta P_{\text {loss }}\left(V_{D D}^{*}, V_{D D}^{* N}, d\right)$ is calculated by

$$
\begin{aligned}
& \operatorname{Exp}\left(\Delta P_{\text {loss }}\right)=\operatorname{Exp}\left(\Delta P_{\text {loss }}\left(V_{D D}^{*}, V_{D D}^{* N}, d\right)\right)= \\
& \sum_{j=L_{0}}^{L_{\max }}\left\{\sum_{k=L_{0}}^{\bar{j}} \sum_{l_{k}=0}^{N_{\text {pixel }}-N_{\text {dist }}}\left[\Delta P_{\text {loss }}\left(V_{D D}^{*}, V_{D D}^{* N}, d\right)\right) \cdot l_{k} \cdot \operatorname{Pr}\left(l_{k}, j\right)\right] \\
& \left.+\sum_{k=\bar{j}}^{L_{\max }} \sum_{l_{k}=0}^{N_{\text {dist }}}\left[\Delta P_{\text {loss }}\left(V_{D D}^{*}, V_{D D}^{* N}, 1\right) \cdot l_{k} \cdot \operatorname{Pr}\left(l_{k}, j\right)\right]\right\} .
\end{aligned}
$$

where $\bar{j}$ represents the corresponding $L_{T}^{N}$ value for $j$, which is given by

$$
\bar{j}=\left\lceil\frac{j}{\left(L_{\max } / n\right)}\right\rceil \cdot \frac{L_{\max }}{n},
$$

and the number of distorted pixel are calculated by

$$
N_{\text {dist }}=\sum_{i=\bar{j}}^{L_{\max }} \mathcal{H}_{L}(i) .
$$

We calculated $d, V_{D D}^{*}$, and $V_{D D}^{* N}$ from (3), (5), (6) and (7). We denote the probability such that $k$-th interval in the histogram without quantization has $l_{k}$ pixels where $L_{T}=j$ by $\operatorname{Pr}\left(l_{k}, j\right)$, which is given by

$$
\begin{aligned}
& \operatorname{Pr}\left(l_{k}, j\right)=\operatorname{Pr}\left(\mathcal{H}_{L}(k)=l_{k} \mid L_{T}=j\right) \\
& =\left\{\left(\begin{array}{c}
N_{\text {pixel }} \\
N_{T}
\end{array}\right)\left(\frac{L_{\max }-j}{L_{\max }}\right)^{N_{T}}\left(1-\frac{L_{\max }-j}{L_{\text {max }}}\right)^{N_{\text {pixel }}-N_{T} .}\right. \\
& \left.\left(\begin{array}{c}
N_{\text {pixel }}-N_{T} \\
l_{k}
\end{array}\right)\left(\frac{1}{L_{\text {max }}}\right)^{l_{k}}\left(1-\frac{1}{L_{\text {max }}}\right)^{N_{\text {pixel }}-N_{T}-l_{k}}\right\},
\end{aligned}
$$

where $N_{\text {pixel }}$ is the total number of OLED cells on the panel, $N_{T}$ is the number of pixels that have higher luminance than $L_{T}$, and the value of $L$ is uniformly distributed from $L_{0}$ to $L_{\max }$. Without loss of generality, each binomial probability mass function component in (16) can be approximated to the normal distribution because $N_{\text {pixel }}$ is large enough and $N_{\text {pixel }} \cdot\left(1 / L_{\text {max }}\right)$ is far greater than 10 as far as $N_{T}$ is smaller than $10 \%$ of $N_{\text {pixel }}$.

We obtain the expected power saving with $n$-interval histogram by

$$
\begin{aligned}
\operatorname{Exp}\left(P_{\text {saving }}\right)= & \operatorname{Exp}\left(\Delta P_{\text {loss }}\left(\max \left(V_{D D}\right), V_{D D}^{*}, d\right)\right. \\
& -\operatorname{Exp}\left(\Delta P_{\text {loss }}\left(V_{D D}^{*}, V_{D D}^{* N}, d\right),\right.
\end{aligned}
$$

where the $\max \left(V_{D D}\right)$ is $15 \mathrm{~V}$ which is used in the implementation. Each histogram interval requires a counter register that can accommodate $N_{\text {pixel }}$. We consider the overhead of counters and calculate $P_{\text {saving }}$ per counter, $P_{\text {saving }} / n$, to consider resource complexity. Fig. 12 shows $\operatorname{Exp}\left(\Delta P_{\text {loss }}\right)$ for different $n$ 


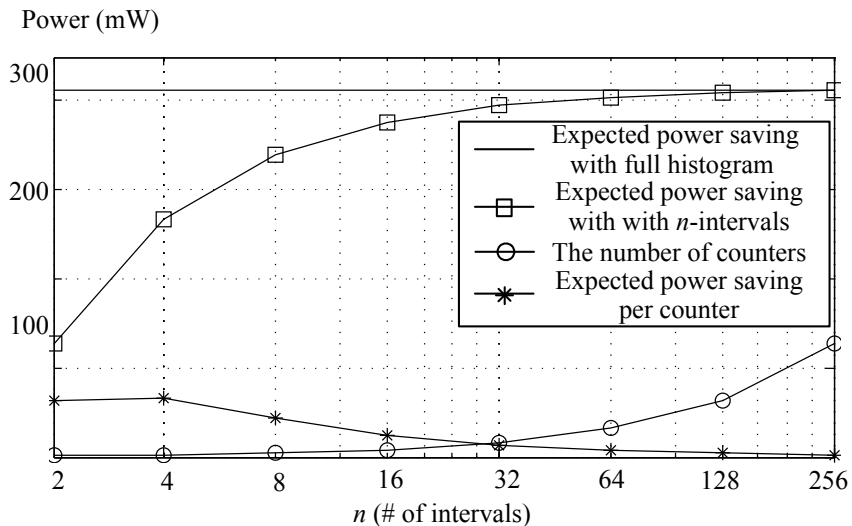

Fig. 12. $P_{\text {saving }}$ vs. the number of counters.

in a $220 \times 176$ display panel with $\tau_{\text {image }}$ corresponding to the $5 \%$ of totally distorted pixels. As shown in Fig. 12, $P_{\text {saving }} / n$ decreases as $n>4$. We confirm that 4-step histogram shows the most efficient results for the target OLED system.

\section{B. $V_{D D}$ transition overhead}

Transition energy overhead in a DVS-enable system was carefully studied in [20]. When $V_{D D}$ range is from $8 \mathrm{~V}$ to $15 \mathrm{~V}$ with a $22 \mu \mathrm{F}$ output bulk capacitor in the DC-DC converter (which is used in our implementation), the required energy to charge the output bulk capacitor is about $0.76 \mathrm{~mJ}$. The maximum voltage transition frequency is determined by the refresh rate of the OLED panel. If we use $60 \mathrm{~Hz}$ refresh rate, then the expected power overhead for the voltage transition is about $46 \mathrm{~mW}$. However, as indicated in [20], the extra energy consumed as a result of scaling up $V_{D D}$ can be compensated for by the $V_{D D}$ downscaling. Consequently, the expected amount of wasted energy is negligible for random $V_{D D}$ change.

Modern DC-DC converter requires for a few tens of $\mu$ s for a voltage transition within the operating range [20]. OLED display panels have idle intervals during VSYNC period. The length of each VSYNC period is typically about 3 HSYNC cycles, and the HSYNC is asserted at the end of horizontal scan. Consequently, VSYNC period is about 9 horizontal scan cycles. The length of data transfer cycle is determined by the refresh rate and the display size. If we use $60 \mathrm{~Hz}$ refresh rate in 1920 x 1200 display, then the idle period between the refresh operation is about $130 \mu \mathrm{s}$. Therefore, the voltage transition is feasible during the idle period even when we use a large-size panel.

\section{IMPLEMENTATION}

\section{A. Hardware implementation}

We develop an hardware board which enables $V_{D D}$ scaling and the image compensation for the target OLED display panel. This platform equips two output voltage adjustable DC-DC converters for an UG-2076 OLED display panel. We modify the output voltage feedback loop of a LT3495 DC-DC
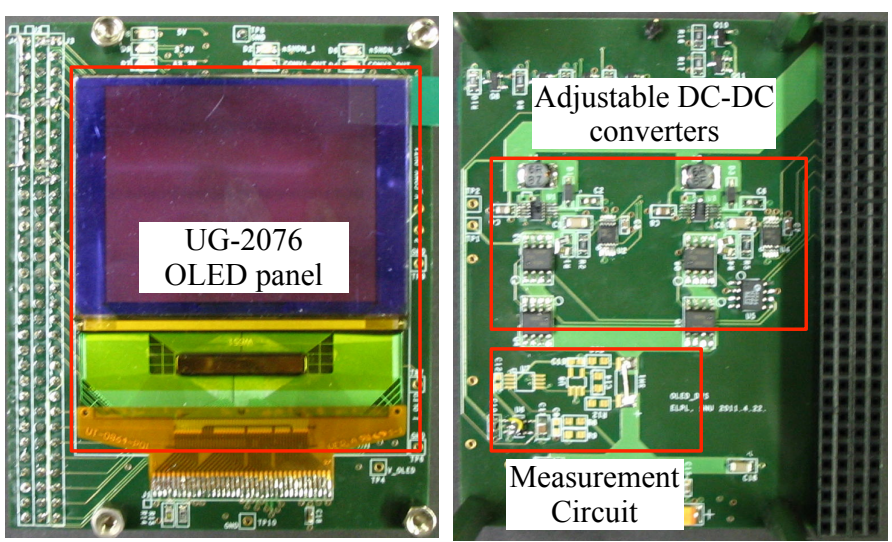

Fig. 13. Output voltage adjustable DC-DC converter equipped OLED display board.

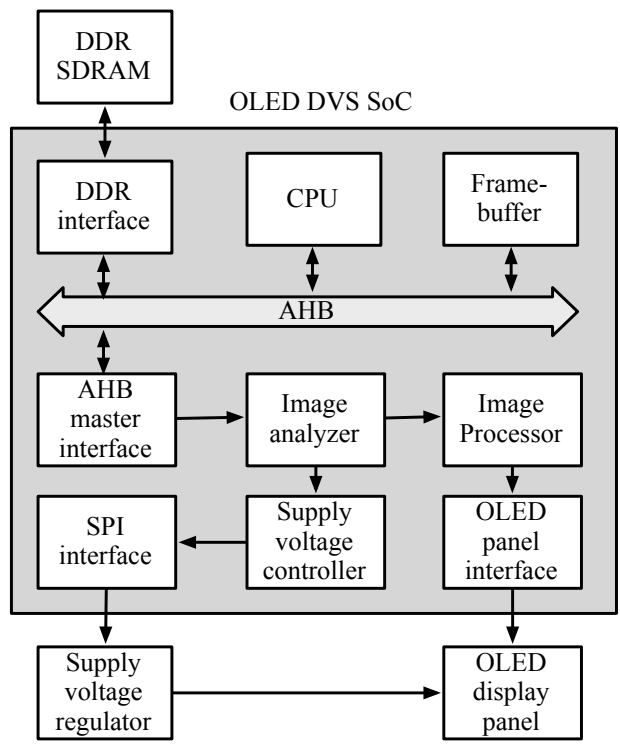

Fig. 14. OLED DVS enabled system-on-chip implemented with an FPGA.

converter form Linear Technology by using an AD5161 digital potentiometer from Analog Device. The platform is compatible with the peripheral interface of a Xilinx Virtex-5 FPGA-based evaluation platform. We control the OLED display panel and power converters through the FPGA platform. Supply voltage and current are measured by an INA194 current shunt monitor form Texas Instrument and an ADC102S Analog-Digital converter from National Semiconductor.

\section{B. OLED DVS System-on-Chip}

We implement an OLED DVS system-on-chip (SoC) on a FPGA as shown in Fig. 14. The SoC consists of an ARM7 microprocessor, a DDR SDRAM interface, an on-chip SRAM framebuffer, an image analyzer, an image processor, a $V_{D D}$ controller, and a SPI interface. The microprocessor transfers the image data to the framebuffer, and the AHB master interface provide the frame data to the OLED display panel interface according to the sweep rate of the OLED display panel. The image analyzer finds the optimal $V_{D D}$ and a lookup table map of image data by constructing the histogram of the pixel data. According to the value obtained by the image analyzer, the $V_{D D}$ controller adjusts the output voltage of 
TABLE III

SYNTHESIS RESULT AND POWER MEASUREMENT OF OLED DVS UNIT IN FPGA.

\begin{tabular}{|c|c|c|c|}
\hline & & Original & OLED DVS \\
\hline \multirow{3}{*}{$\begin{array}{c}\text { Synthesis } \\
\text { result }\end{array}$} & Slices & 8353 & 8993 \\
\cline { 2 - 4 } & Slice Reg. & 7910 & 8131 \\
\cline { 2 - 4 } & LUTs & 13261 & 14629 \\
\cline { 2 - 4 } & LUTRAM & 1109 & 1109 \\
\cline { 2 - 4 } & BRAM & 130 & 130 \\
\hline \multicolumn{2}{|c|}{ Power consumption } & $6.273 \mathrm{~W}$ & $6.319 \mathrm{~W}$ \\
\hline
\end{tabular}

the $V_{D D}$ regulator through the SPI interface, and the image processor modifies the pixel data synchronized with each other.

The image analyzer builds a luminance histogram of the image for each color. The image analyzer consists of pixel counter and registers storing threshold values. The number of counters accruing to the histogram steps in the image analyzer is synthesizable, and the threshold values are programmable by user. We separately setup the threshold values in the linear region and non-linear region according to the relation between the luminance and $V_{D D}$ as shown in Fig. 7. We estimate the image distortion from the image histogram and select the $V_{D D}$ level. The image processor consists of arithmetic operators and lookup table. The image data output value is calculated by a piece-wise linear model with the coefficients stored in the lookup table. The number of piece in the model is synthesizable, and the lookup table is programmable.

The OLED DVS unit accepts the $V_{D D}$ level and original image data as an input, and generates a modified image data value to compensate the effect of $V_{D D}$ scaling. The $V_{D D}$ controller adjusts the output voltage of the DC-DC converter by changing the value of feedback resistance. We connect the digital potentiometer to the feedback input of the DC-DC converter. The resistance value of the digital potentiometer can be controlled by a serial peripheral interface (SPI). The $V_{D D}$ controller transmits SPI signal synchronized with a frame synchronization signals.

Table III summarizes space complexity of the DVS-enabled OLED display panel controller synthesized in the FPGA. We measure the average power consumption of the Xilinx XUPV5-LX110T platform when the OLED DVS module is on and off with the same image sequence. The difference between the power consumption of OLED DVS enabled and disabled display controller is $46 \mathrm{~mW}$ that corresponds to $0.73 \%$ of the total power consumption. The synthesis result shows that the OLED DVS enabled system requires $7.66 \%$ more slices (CLBs).

We also estimate the area and power overhead for an ASIC implementation. We obtain the silicon area and estimated power consumption value for the OLED DVS controller unit by using TSMC $45 \mathrm{~nm}$ technology with Synopsys Design Compiler. The results are summarized in Table IV.

\section{EXPERIMENTS}

\section{A. Simulation result for OLED display with AM driver}

We implement a prototype by using an OLED display panel which equips PWM driver circuit. We perform a simulation
TABLE IV

SILICON AREA AND ESTIMATED POWER CONSUMPTION OF OLED DVS UNIT WITH TSMC 45 NM TECHNOLOGY.

\begin{tabular}{|c|c|}
\hline \multicolumn{2}{|l|}{ Area } \\
\hline Number of nets & 2274 \\
\hline Number of cells & 173 \\
\hline Number of references & 41 \\
\hline Combinational area & 3667.179605 \\
\hline Noncombinational area & 1181.527204 \\
\hline Total cell area & 4848.706809 \\
\hline \multicolumn{2}{|c|}{ Power consumption } \\
\hline Global operating voltage & 0.99 \\
\hline Cell internal power & $199.0935 \mathrm{uW}$ \\
\hline Net wwitching power & $72.8849 \mathrm{uW}$ \\
\hline Total dynamic power & $271.9784 \mathrm{uW}$ \\
\hline Cell leakage power & $297.7593 \mathrm{uW}$ \\
\hline
\end{tabular}

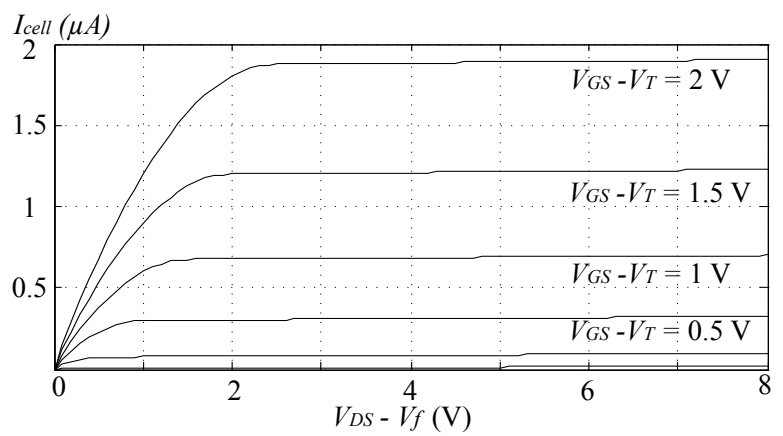

Fig. 15. TFT driver gate characteristics for simulation of OLED panel with AM driver.

to evaluate the effect of OLED DVS with AM driver-based OLED display. Fig. 15 shows the I-V characteristics of the driver gate. Based on the driver gate characteristics presented in Fig. 15 and optical characteristics presented in Section IV-B, we evaluate the OLED DVS with several standard test images. Fig. 16 shows the result for a Lena, a mandrill, a boat and an airplane. We use the characterization result presented in Section IV-B to calculate the power consumption and image distortion. The images in first row in Fig. 16 are original test images. The second row is $V_{D D}$-scaled images constrained by the number of distorted pixels. We limit the number of distorted pixels within 5\%. The average power saving is $37 \%$.

As described in Section IV-C we cannot compensate the image distortion with the AM driver circuit due to the current mirror operation in the saturated region. Therefore, the distortion of bright pixel is not avoidable. We show the location of distorted pixels in the last row of Fig. 16. The distorted pixels are represented by color inversion. We can see that the bright parts of the images are distorted.

We evaluate the histogram-based OLED DVS by simulation. Fig. 17 shows the $V_{D D}$ scaling and estimated power result for the movie clip. We use 10 seconds of movie clip from the 'How to Train Your Dragon.' The movie clip is carefully chosen to contain bright scenes and dart scenes keeping the balance. $V_{D D}$ is scaled up to $9 \mathrm{~V}$ while play the movie clip. The original movie clip with $15 \mathrm{~V} V_{D D}$ shows $5.45 \mathrm{~J}$ energy consumption during $10 \mathrm{~s}$, and the movie clip with a $5 \%$ dis- 


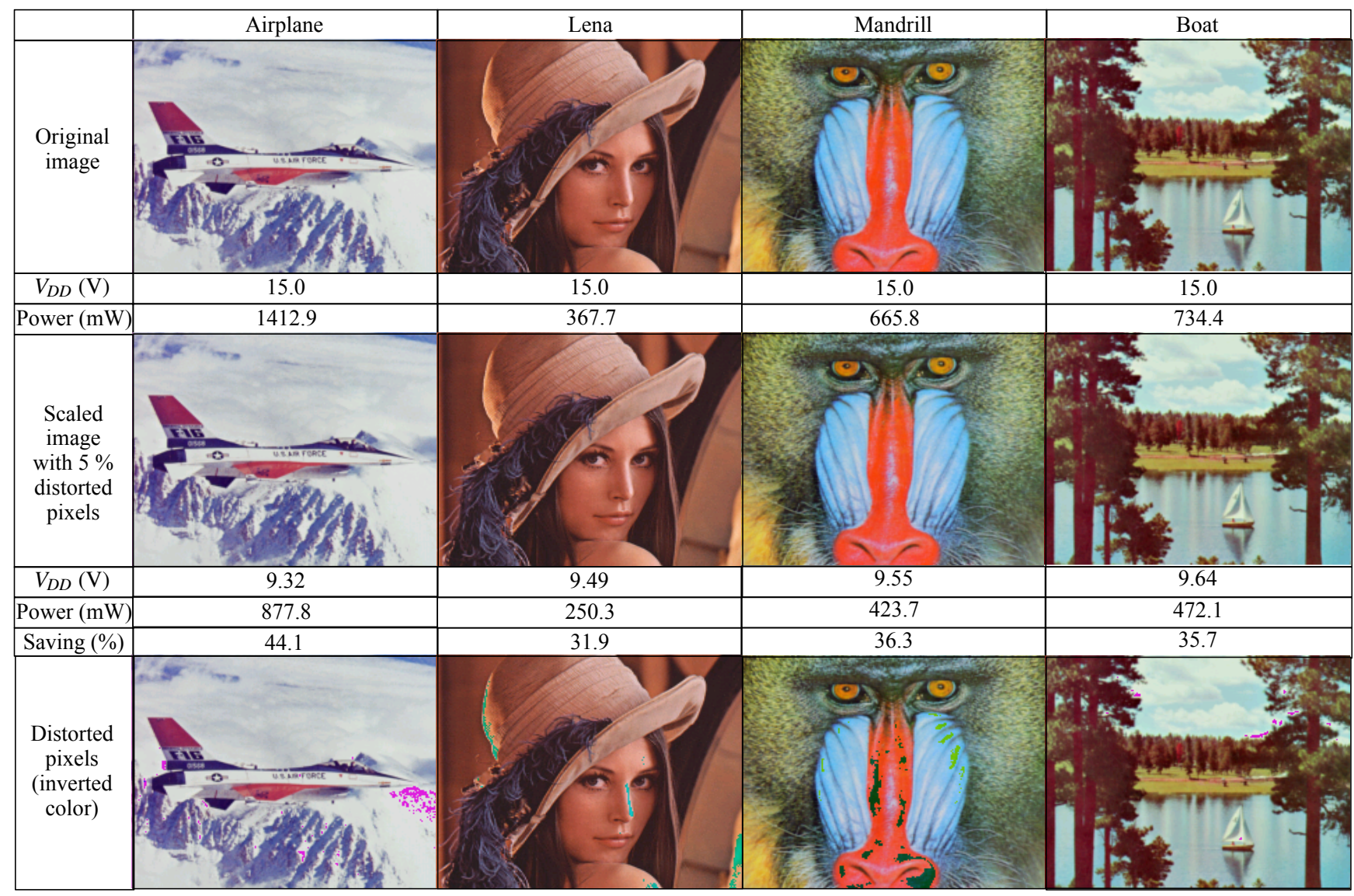

Fig. 16. Simulation results for AM driver-based OLED display with standard test images.

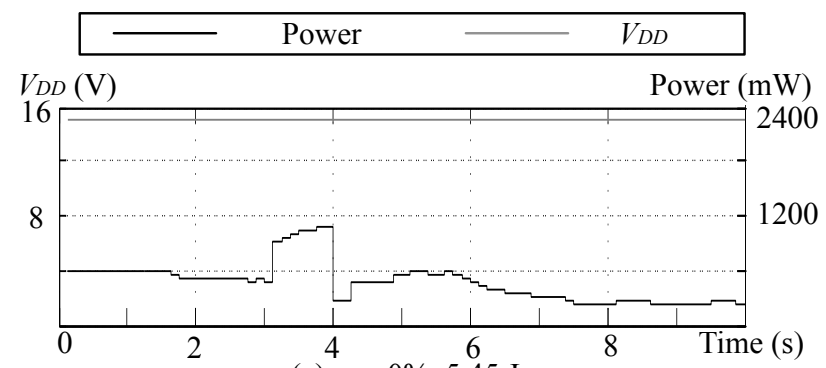

(a) $\varepsilon=0 \%, 5.45 \mathrm{~J}$

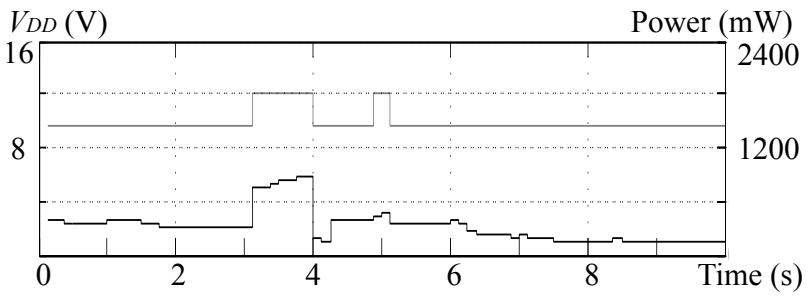

(b) $\varepsilon=5 \%, 3.54 \mathrm{~J}$

Fig. 17. Simulation results of power consumption with OLED DVS for the movie clips with (a) $0 \%$ distortion tolerance (b) $5 \%$ distortion tolerance.

tortion constraint shows $3.54 \mathrm{~J}$.

\section{B. Measurement result for OLED display with PWM driver}

We evaluate the power gain and resultant image quality from the proposed OLED DVS on real still images and image

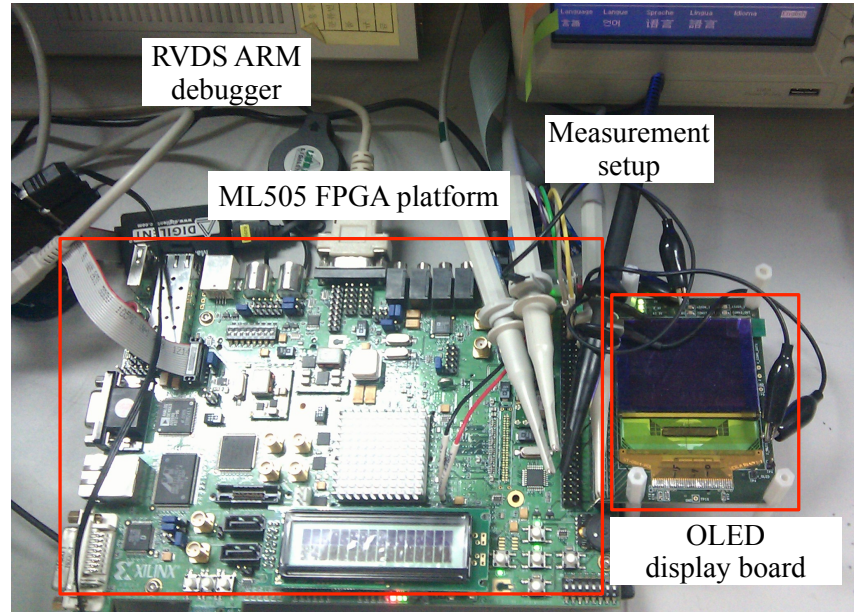

Fig. 18. Experimental setup for the OLED DVS with the prototype implementation.

sequence from a movie clip. Fig. 18 shows the experimental setup with the implemented prototype.

Fig. 19 summarizes the result for the still images. We capture the displayed images on the target OLED display panel by digital camera. The Lena and mandrill images have a typical balanced color distribution while the boat and 


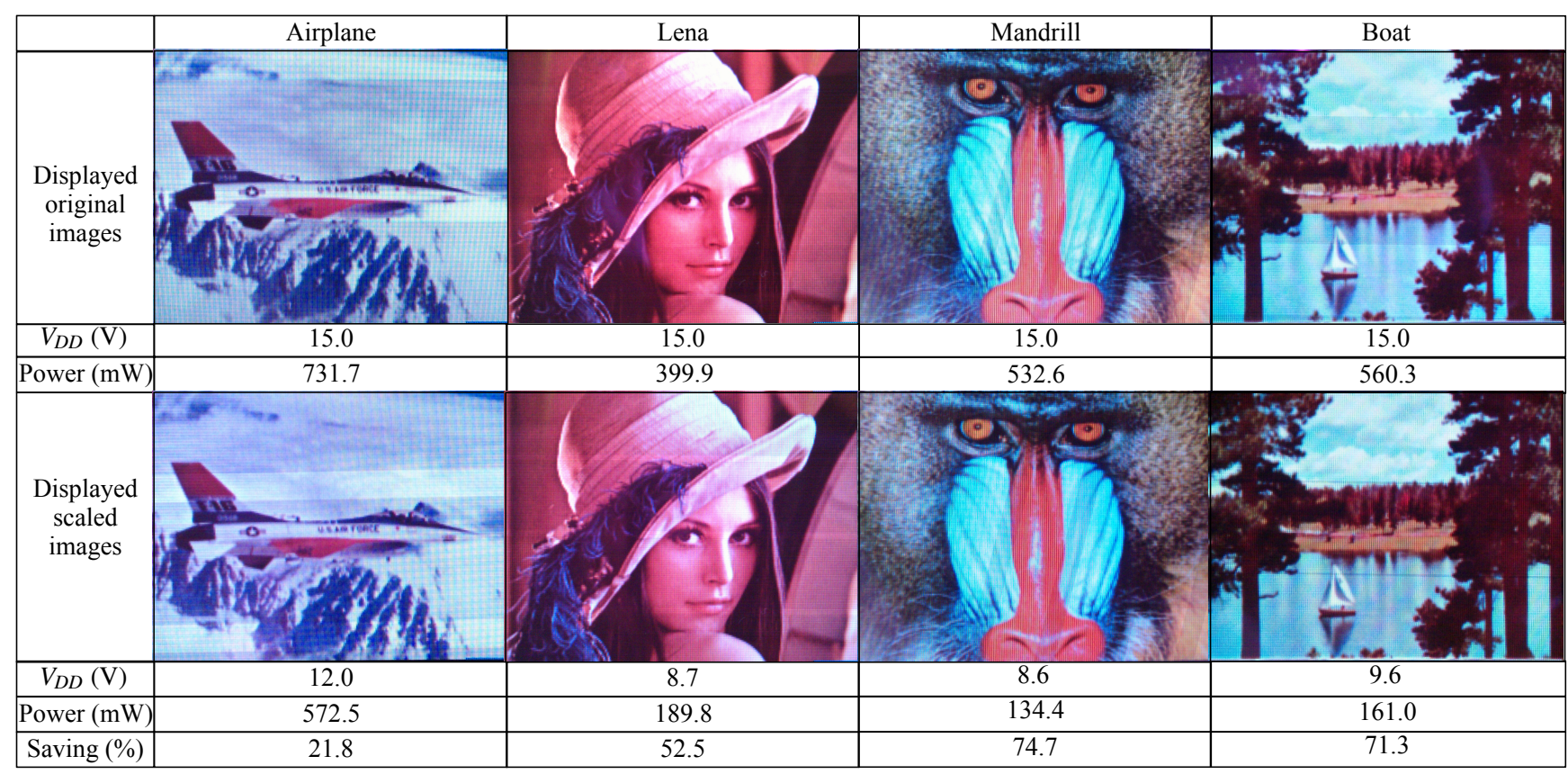

Fig. 19. Photographs of the actual image on the OLED panel in PWM driver equipped prototype with standard test images.

airplane images have a severe skew toward the bright colors, which is challenging for the OLED DVS. The originally high luminance pixels are saturated to the maximum luminance as shown in the compensated images and histograms. The saturated pixels result in the image distortion, but the overall image quality is not appreciably altered within the threshold value.

The Lena image shows $52.5 \%$ power saving compared with the original image while $V_{D D}$ being scaled down from $15 \mathrm{~V}$ to $8.7 \mathrm{~V}$ and with nearly zero color distortion. The mandrill image shows $74.7 \%$ power saving while $V_{D D}$ being scaled down from $15 \mathrm{~V}$ to $8.6 \mathrm{~V}$ and with 300 as the average distortion. The boat image shows $71.3 \%$ power saving $V_{D D}$ being scaled down from $15 \mathrm{~V}$ to $9.6 \mathrm{~V}$ and with 300 as the average distortion. As for the worst case among the benchmarks, the airplane still exhibits $21.8 \%$ power saving compared to the original image with $15 \mathrm{~V} V_{D D}$.

We measure the display power consumption while playing the same movie clip in Section VII-A to evaluate the prototype and SoC. As shown in Fig. 20, the original movie clip with a $15 \mathrm{~V} V_{D D}$ shows $7.44 \mathrm{~J}$ energy consumption during $10 \mathrm{~s}$, and a movie clip with a 5\% distortion constraint shows $4.77 \mathrm{~J}$. We confirm that $V_{D D}$ is more aggressively scaled with larger distortion threshold in Fig. 20.

\section{CONCLUSION}

Organic light emitting diode (OLED) panels are promising display devices capable of self-illumination and thus exhibiting high power efficiency. However, OLED panel consumes comparable amount of power to liquid crystal display (LCD) due to serious total internal reflection. All previous OLED power saving methods change the pixel colors since the pixel color determines the OLED power consumption. Unfortunately, these methods result in significant degradation of the image.

This paper presents the OLED power saving method that enables only minimal pixel distortion, small enough to work with natural images. Furthermore, the proposed technique can be applied to most OLED panel structures. We developed such a unique power saving technique based on a careful analysis of the OLED driver architectures. The proposed method is called OLED dynamic voltage scaling (OLED DVS). We scale down the supply voltage and, in turn, dramatically reduce the wasted power caused by the voltage drop across the driver transistor as well as internal parasitic resistance. The proposed OLED DVS may incur image distortion after the supply voltage scaling. In this case, we compensate the image data based on the humanperceived color space.

We present a simulation result for the OLED panel with the AM driver circuit. We implemented a prototype with the PWM driver equipped-panel and demonstrate the OLED DVS. We demonstrated the OLED DVS for the still images with a prototype implementation and confirmed a $74.7 \%$ power saving for the Lena image with virtually zero distortion. We also measure the power saving of the OLED DVS for the sample movie clips by the using the prototype, and confirm up to $35.9 \%$ display power saving in average with $0.7 \%$ power and $7.7 \%$ slice overhead on the prototype implementation.

\section{REFERENCES}

[1] D. Shin, Y. Kim, N. Chang, and M. Pedram, "Dynamic voltage scaling of OLED displays," in the Annual Conference on Design Automation (DAC), June 2011.

[2] N. Chang, I. Choi, and H. Shim, "DLS: Dynamic backlight luminance scaling of liquid crystal display," IEEE Transactions on Very Large Scale Integration Systems (TVLSI), vol. 3, no. 8, pp. 837-846, 2004. 


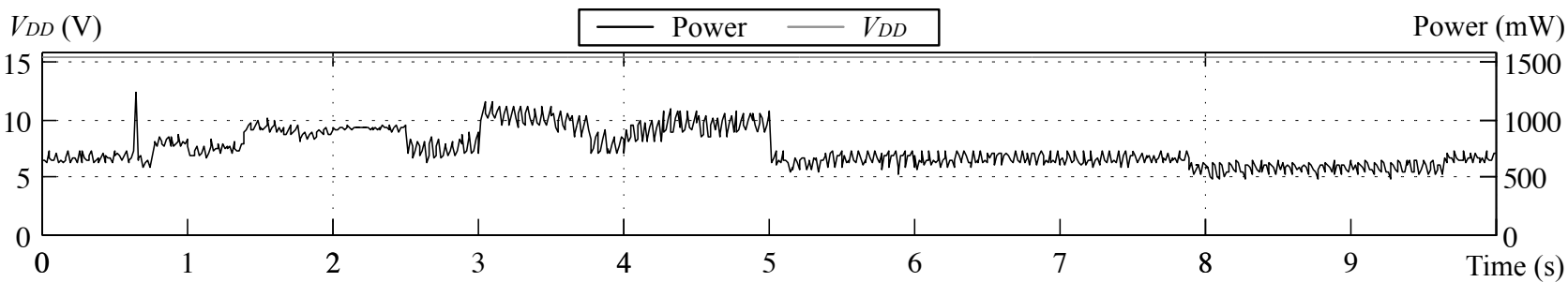

(a) $\varepsilon=0 \%, 7.44 \mathrm{~J}$

$V_{D D}(\mathrm{~V})$

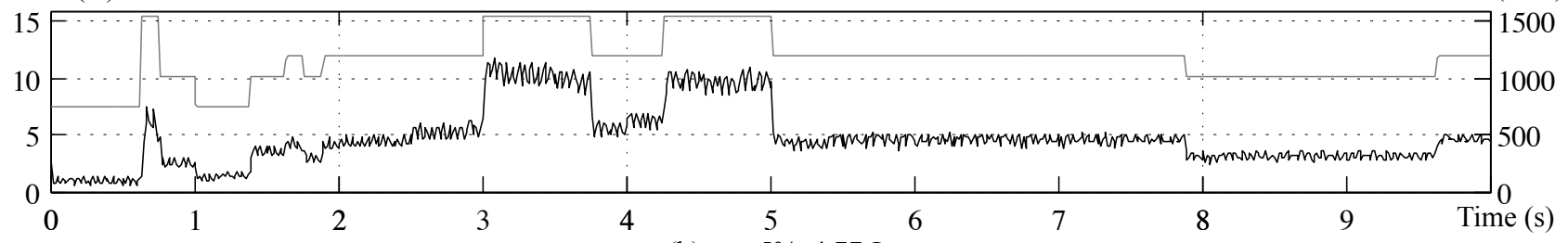

(b) $\varepsilon=5 \%, 4.77 \mathrm{~J}$

Fig. 20. Measured power consumption with OLED DVS for the movie clips with (a) $0 \%$ distortion tolerance (b) $5 \%$ distortion tolerance

[3] H. Shim, N. Chang, and M. Pedram, "A backlight power management framework for battery-operated multimedia systems," IEEE Design and Test of Computers (DATC), vol. 21, no. 5, pp. 388-396, 2004.

[4] J. Jacobs, D. Hente, and E. Waffenschmidt, "Drivers for OLEDs," in IEEE Industry Applications Conference, pp. 1147 -1152, 2007.

[5] A. B. Dalton and C. S. Ellis, "Sensing user intention and context for energy management," in USENIX Workshop on Hot Topics in Operating Systems (HotOS), pp. 26-26, 2003.

[6] V. Moshnyaga and E. Morikawa, "LCD display energy reduction by user monitoring," in IEEE International Conference on Computer Design (ICCD), pp. $94-97,2005$.

[7] J. Flinn and M. Satyanarayanan, "Energy-aware adaptation for mobile applications," in ACM Symposium on Operating Systems Principles (SOSP), pp. 48-63, 1999.

[8] J. Betts-LaCroix, Selective Dimming of OLED displays. US Patent 0149223 A1, 2010.

[9] I. Choi, H. S. Kim, H. Shin, and N. Chang, "LPBP: low-power basis profile of the Java 2 micro edition," in IEEE/ACM International Symposium on Low Power Electronics and Design (ISLPED), pp. 36-39, 2003.

[10] M. Dong, Y.-S. K. Choi, and L. Zhong, "Power-saving color transformation of mobile graphical user interfaces on OLED-based displays," in IEEE/ACM International Symposium on Low Power Electronics and Design (ISLPED), pp. 339-342, 2009.

[11] P. Ranganathan, E. Geelhoed, M. Manahan, and K. Nicholas, "Energyaware user interfaces and energy-adaptive displays," Computer, vol. 39, no. 3, pp. $31-38,2006$.

[12] I. Choi, H. Shim, and N. Chang, "Low-power color TFT-LCD display for hand-held embedded systems," in IEEE/ACM International Symposium on Low Power Electronics and Design (ISLPED), pp. 112 - 117, 2002.

[13] F. Gatti, A. Acquaviva, L. Benini, and B. Ricco', "Low power control techniques for TFT-LCD displays," in IEEE International Conference on Automation Science and Engineering, pp. 218-224, 2002.

[14] W.-C. Cheng, Y. Hou, and M. Pedram, "Power minimization in a backlit TFT-LCD display by concurrent brightness and contrast scaling," in Design Automation and Test in Europe, vol. 1, pp. 252 - 257, 2004.

[15] W.-B. Lee, K. Patel, and M. Pedram, "White-LED backlight control for motion-blur reduction and power minimization in large LCD TVs," Journal of the Society for Information Display, vol. 17, no. 1, pp. 37 45, 2009.

[16] X. Chen, J. Zheng, Y. Chen, H. Li, W. Zhang, S. wei Liao, and J. Wang, "Fine-grained dynamic voltage scaling on OLED display," in IEEE Asia and South Pacific Design Automation Conference (ASPDAC), 2012.

[17] B. Fraser, C. Murphy, and F.Bunting, Real world color management. Pearson Education, 2002.

[18] A. K. Jain, Fundamentals of Digital Image Processing. Prentice hall, 1988.

[19] UG-2076GDEAF02 OEL Display Module Product Specification. Univision Technology Inc.
[20] J. Park, D. Shin, M. Pedram, and N. Chang, "Accurate modeling and calculation of delay and energy overheads of dynamic voltage scaling in modern high-performance microprocessors," in IEEE/ACM International Symposium on Low Power Electronics and Design (ISLPED), pp. 419424, August 2010. 